\title{
Gedacht soll seiner werden: Dr. Ernst Weiß, 28. August 1882 bis 15. Juni 1940
}

Enrico Danieli

Korrespondenz:

Dr. med. Enrico Danieli

Via ai Colli 22

CH-6648 Minusio
«Dr. Ernst Weiß hat sich in Paris das Leben genommen, in der Stadt, die dem mehrfach Vertriebenen ein drittes und letztes Asyl gab, im kleinen Hotel an der Avenue de Versailles, in dem er die letzten Jahre seines Emigrantendaseins fristete - seine Bücher werden noch gelesen werden, wenn die Deutschen längst vergessen haben, wie der Autor hat leben müssen, wie er gestorben ist» (Nachruf W. Bredel 1941).

Ernst Weiß, Arzt und Schriftsteller, stirbt ein paar Stunden nach dem Einmarsch deutscher Truppen in Paris. Er, der von sich sagte, er habe immer Unglück im Leben gehabt, hatte es bis zuletzt, ein deutsch-jüdischer Dichter aus «Alt»Österreich zur Zeit von Deutschlands tiefster Schmach. Einer, der ein doppeltes Kreuz zu tragen hat. Nach dem Ende des Krieges will man von ihm nichts mehr wissen, Emigrantenautoren werden aus dem Gedächtnis gelöscht. «Gedacht soll ihrer werden» (Adorno).

E. W. wird 1882 in Brünn geboren, sein Vater, Tuchhändler, stirbt 1886, der Sohn studiert Medizin in Berlin und Bern (bei Theodor Kocher), wo er sich zum Chirurgen ausbilden lässt. Später heuert er als Schiffsarzt an und bereist den Fernen Osten. Im ersten Krieg wird er an der Front wie im Hinterland als Arzt eingesetzt. Ab den 20er Jahren in Berlin, widmet er sich einzig dem Schreiben. Es folgen Romane, Erzählungen (z.B. «Franziska», 1916) und Essays, in literari-

«Genieße doch, was du hast! Arzt sein ist kein Genuß. Es ist der undankbarste Beruf, den es gibt. Mit Kranken und Unheilbaren sein Leben zu vertrödeln, trotz allem Schweiß und aller Mühe niemals mehr als nur einen Fingerbreit aufzubauen und zum Schluß doch in der Anatomie alles enden sehen unser Werk hat eben keinen Bestand.» «Aber es muß doch Ärzte geben! Was sollte denn sonst aus uns werden?» fragte ich voll Angst. «Ob es sich lohnt, frage ich mich oft. Wenn es zu klagen und zu jammern gilt, dann übertreiben die Menschen, diese ordinäre Horde. Sind sie aber geheilt, dann soll es nichts gewesen sein. Ein Kaufmann hat, was er ver- schen Kreisen wird E. W. allmählich anerkannt. In Paris lebt er ab Mitte der 30er Jahre, hier entstehen die grossen Romane: «Der arme Verschwender» (1936), Stefan Zweig gewidmet, der ihn finanziell unterstützt, «Der Verführer» (1938), Thomas Mann gewidmet, sowie - aktueller denn je-der Hitler-Roman «Der Augenzeuge» aus dem Jahre 1939 (Dieses Manuskript taucht erst 1963 auf: Der Roman wird postum veröffentlicht und wird Ernst Weiß' bekanntester: die Geschichte eines Arztes, der gegen Ende des ersten Krieges den psychisch gestörten A. H. behandelt und damit dessen spätere Karriere ermöglicht ...). Seine Schreibkraft nimmt der Autor aus seinem Wissen um die Psychoanalyse Sigmund Freuds es lässt sich behaupten, dass es ihm gelingt, das Wesen der Neurose mit ihren verheerenden Folgen romanhaft darzustellen - und, wie er selbst betont, aus den exakt beschreibenden Krankengeschichten Theodor Kochers, seines Lehrers in Bern. «Durch ihn lernte ich sehen und schildern und erkennen.»

Die Kriegserlebnisse finden Widerhall in mehreren Romanen (z.B.: «Georg Letham. Arzt und Mörder», 1931). - «Mich trifft das Wort Vater wie ein Hufschlag»: Das Pubertätsdrama - zwischen «Törleß» und «Unterm Rad» - «Der Aristokrat» (1926) zeigt den zukünftigen Weg auf: Vaterbindungen (die im Grunde genommen, so der Autor, immer unerfüllte Liebesgeschichten sind)

dient. Er hat es sich erworben, es ist sein. Niemand bestreitet es ihm. Aber hat unsereins etwas geleistet, dann sind nicht wir es gewesen, es ist die Heilkraft der Natur gewesen. Ist aber etwas mißlungen, so war es deine Schuld.» «Nein, das glaube ich nicht», unterbrach ich inn leidenschaftlich, «niemals!» «So, das glaubst du nicht? Ich aber weiß es. Es ist vielleicht ein edler Beruf, den Leidenden zu helfen, wirst du großer Junge mit deinem Vorzugszeugnis sagen» - ich nickte lebhaft -, «aber es ist und bleibt ein erbärmlicher Broterwerb.»

Ernst Weiß: Der arme Verschwender (1936) 
und Todesangst (die nichts anderes ist, schreibt der Autor, als Furcht vor dem Leben). In den beiden grossen noch zu Lebzeiten des Autors veröffentlichten Romanen, beide in Ich-Form erzählt, sucht der vom Faschismus Vergetriebene Zuflucht in der Vergangenheit, in seinem «unvergesslichen» Österreich-Ungarn. Im Roman «Der arme Verschwender» - vergleiche Kasten rühmt Thomas Mann die bewundernswerte künstlerische Diskretion und die melancholische Tiefe des Lebensgefühls: Es geht um die erbarmungslose Zerstörung eines Sohnes durch seinen Vater, berühmter Augenarzt und Dozent. Der Sohn, der dem Vater immer zu Diensten steht, ebenso Augenarzt wird, wird zum Opfer eines unvermeidlichen Untergangs, ohne dass er sich selbst dessen bewusst ist: ein auch bis heute aktuelles Musterbeispiel schwarzer Pädagogik. Ein Sohn, der stets sein Bestes tut und (deswegen) scheitert. Unbeschwerter der Roman «Der Verführer»: Wiederum steht im Zentrum eine vergötterte, ehrbare, mächtige Vaterfigur, diese bestimmt noch aus dem Grab heraus des Sohnes Leben. Der Sohn folgt dem Vater wie an unsichtbaren Fäden gezogen in allem Üblen nach. Ernst Weiß ist keiner der vielen Geschilderten in seinen Büchern - oder alle zugleich. Ernst Weiß, ein grosser Autor, den man nicht, wie viele andere, vergessen, den man nicht auch noch um sein Nach-Leben bringen darf.

\section{Plastische Chirurgie}

\section{S. Krupp, H. O. Rennekampff, N. Pallua}

\section{Plastische Chirurgie - Klinik und Praxis}

Landsberg: ecomed Medizin; 2006

Neuauflage mit 28. Ergänzungslieferung, Loseblattausgabe (Bd. 1-2)

$€ 249$.-

ISBN 978-3-609-76212-8
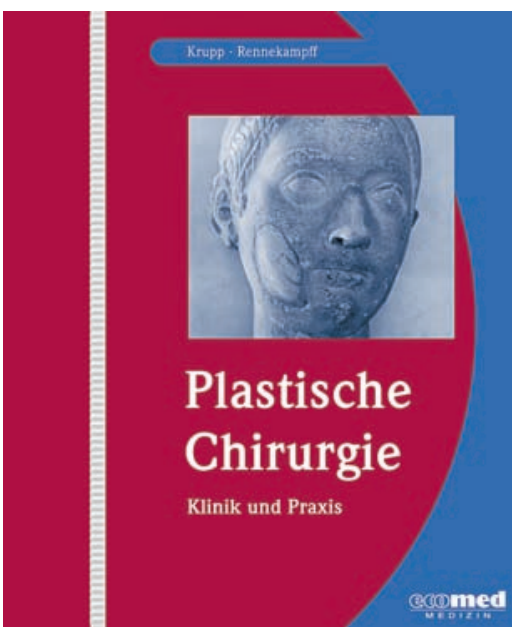

Wenn man «Plastische Chirurgie» hört, denkt man als erstes an «Ästhetische Chirurgie». Dieses Teilgebiet macht jedoch nur ein kleines Kapitel aus. Hinter «Plastischer Chirurgie» verbirgt sich einiges mehr, wie zum Beispiel: Korrektur von Missbildungen, Wundbehandlung und -verschluss, Gewebetransfer, Behandlung von Verbrennungen, Mikrochirurgie sowie Handchirurgie. So ist die Plastische Chirurgie zwar ein eigenständiges Fachgebiet, das jedoch Verbindungen $\mathrm{zu}$ allen anderen chirurgischen Teilgebieten aufrechterhält.

Bereits 1994 ist die Erstauflage dieses Werkes «Plastische Chirurgie» im ecomed-Verlag erschienen. Es ist als Loseblattsammlung konzipiert. Diese Art des Buches macht es möglich, trotz der ständigen Weiterentwicklungen des Faches stets auf dem neuesten Stand zu bleiben. Der Verlag bietet ein Abonnement an, um die Ergänzungslieferungen, die alle sechs Monate erscheinen, zu erhalten. Diese sind anhand der mitgesandten Sortieranleitungen und sich auf jeder Seite befindenden Kapitelnummern leicht einzuordnen. Die Aktualität ist also gewährleistet, zumal separate $\mathrm{Ab}$ schnitte «Ergänzungslieferungen» und «Aktuelles» vorgesehen sind. Der einzige Nachteil des Heftsystems liegt in der schwachen Papierqualität, wobei durch den enormen Umfang des Werkes, trotzdem es schon zweibändig vorliegt, die Löcher nach mehrmaligem Umblättern leicht ausreissen. Ausserdem wurde nicht viel Platz zum Einheften der Ergänzungslieferungen gelassen, so dass bald auf zusätzliche Ordner ausgewichen werden muss.

Schon die Einführung in das Werk ist sehr interessant gestaltet, da sie auf die Geschichte der Plastischen Chirurgie zurückgreift. Ge- mäss den Worten Lissners «Alles historische Geschehen ist unsterblich» wird deutlich, dass Entwicklung und Fortschritt aus Geschichte und Erfahrungen hervorgeht und ohne diese nicht möglich wäre. Man erfährt z.B. einiges über Gelenktransplantationen, was neue Ideen hervorruft und anregt, über aussergewöhnliche Methoden nachzudenken. Berühmte Persönlichkeiten und hervorragende Chirurgen werden mit ihrem Schaffenswerk und ihren Schulen vorgestellt.

Die Monographie, die einzigartig in ihrer Vollständigkeit und ihrem Umfang auf dem Gebiet der Plastischen Chirurgie ist, dient nicht nur als Lehrbuch, sondern ebenso als Nachschlagewerk. Eine Inhaltsübersicht, das ausführliche Inhaltsverzeichnis sowie ein umfangreiches Stichwortverzeichnis helfen beim Aufsuchen spezieller Inhalte. Die Kapitel sind nach anatomischen Gesichtspunkten von Kopf bis zur unteren Extremität gegliedert. Die vielen Fotos und didaktisch ausgezeichneten schematischen Zeichnungen erleichtern das Verständnis. Zum weiteren Vertiefen findet man jeweils am Kapitelende zahlreiche Literaturangaben.

Das Autorenverzeichnis umfasst acht Seiten, was auf die Grösse des internationalen Autorenteams schliessen lässt. Es handelt sich um erfahrene Fachexperten aus ganz Deutschland, der Schweiz, Österreich, Frankreich, Italien, Belgien, Schweden, Norwegen, Israel, Mexiko und den USA.

Sandra Krüger, Lausanne 